\title{
High c-Cbl expression in gliomas is associated with tumor progression and poor prognosis
}

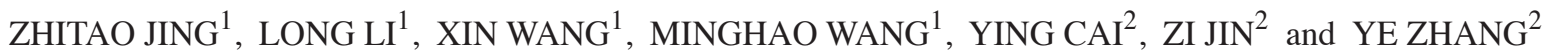 \\ Departments of ${ }^{1}$ Neurosurgery and ${ }^{2}$ Medical Oncology, The First Hospital of China Medical University, \\ Shenyang, Liaoning 110001, P.R. China
}

Received March 10, 2015; Accepted January 26, 2016

DOI: $10.3892 / \mathrm{ol} .2016 .4318$

\begin{abstract}
Casitas B-lineage lymphoma (c-Cbl) expression has been linked to the development of several types of cancer. However, no studies on the association of $\mathrm{c}-\mathrm{Cbl}$ and glioma have been published thus far. The present study examined glioma samples obtained from 136 patients treated at The First Hospital of China Medical University (Shenyang, China) from January 2007 to December 2009, and the expression levels of $\mathrm{c}-\mathrm{Cbl}$ in the samples were evaluated by reverse transcription-quantitative polymerase chain reaction, immunohistochemistry and western blotting. Kaplan-Meier survival curves were generated and subjected to Cox regression analysis. The messenger RNA and protein levels of c-Cbl were observed to be upregulated in high-grade glioma, compared with low-grade glioma. A multivariate analysis revealed that the protein levels of c-Cbl were independently associated with overall survival [hazard ratio $(\mathrm{HR})=4.923,95 \%$ confidence interval $(\mathrm{CI})=3.163-7.662 ; \mathrm{P}<0.001]$. Furthermore, the grade of the glioma (according to the World Health Organization criteria) was observed to be independent prognostic factors for progression-free survival and overall survival time $(\mathrm{HR}=8.842$, 95\% $\mathrm{CI}=7.827-9.989 ; \mathrm{P}<0.001$, and $\mathrm{HR}=10.247$, 95\% CI=9.009-11.655; $\mathrm{P}<0.001$, respectively). Kaplan-Meier analysis and log-rank test indicated that high protein expression levels of c-Cbl were significantly associated with overall and progression-free survival $(\mathrm{P}<0.001)$. To the best of our knowledge, these results provide the first evidence that the overexpression of c-Cbl is correlated with advanced clinicopathological features and poor prognosis in patients with glioma.
\end{abstract}

\section{Introduction}

Gliomas are the most common type of tumors of the primary central nervous system in adults (1). Their highly invasive

Correspondence to: Professor Ye Zhang, Department of Medical Oncology, The First Hospital of China Medical University, 155 North Nanjing Street, Heping, Shenyang, Liaoning 110001, P.R. China E-mail: zhangye@mail.cmu.edu.cn

Key words: c-Cbl, glioma, prognosis, tumor progression nature precludes complete resection, resulting in significant neurological morbidity and mortality (2). Despite progress in surgical, radio- and chemotherapeutic approaches for the treatment of glioma, its prognosis remains poor (3). For instance, patients with glioblastoma multiforme (GBM), which is the most malignant and frequently reported histological type of glioma, present a median life expectancy of only 14.6 months following diagnosis (4). Thus, a greater understanding of the molecular mechanisms of gliomagenesis may lead to more effective, individualized treatments.

Casitas B-lineage lymphoma (c-Cbl) is a really interesting new gene finger-type E3 ubiquitin ligase in the ubiquitin-proteasome pathway (5). Cbl proteins play important roles in the inhibition of growth factor receptors (6). For example, Cbl-mediated ubiquitination of active receptors is essential for their degradation and the termination of receptor-induced signal transduction (7-9). As such, the ubiquitin-proteasome pathway is important for maintaining cellular homeostasis, and mutations in the components of this pathway may result in tumorigenesis (10). Indeed, the expression of $\mathrm{c}-\mathrm{Cbl}$ has been reported to be upregulated in various solid tumors, including gastric carcinoma (11), primary colorectal cancer (12), prostate cancer (13) and non-small cell lung cancer (14).

The association between c-Cbl expression and the clinicopathological features of glioma has not been investigated to date, nor has been the prognostic significance of $\mathrm{c}-\mathrm{Cbl}$ overexpression in this type of tumor. These questions were addressed in the present study by examining the expression levels of $\mathrm{c}-\mathrm{Cbl}$ in samples derived from patients with glioma via reverse transcription-quantitative polymerase chain reaction (RT-qPCR), immunohistochemistry and western blotting, and the correlation between c-Cbl expression, glioma stage and patient survival was assessed.

\section{Materials and methods}

Patients and specimens. The present study protocol was approved by the Ethics Committee of The First Hospital of China Medical University (Shenyang, China). Paraffin-embedded specimens of 136 glioma cases were obtained from The First Hospital of China Medical University from January 2007 to December 2009. The cases comprised 73 men and 63 women, with a mean age of 53.3 years (range, 35-72 years). The clinicopathological features of the study population are summarized 
Table I. Correlation between the protein expression levels of c-Cbl and clinicopathological features of patients with glioma.

c-Cbl protein expression levels

\begin{tabular}{|c|c|c|c|c|}
\hline \multirow[b]{2}{*}{ Variable } & \multirow[b]{2}{*}{$\mathrm{n}$} & & \multirow[b]{2}{*}{ P-value } \\
\hline & & Low $(n=40)$ & High $(n=96)$ & \\
\hline Age (years) & & & & 0.509 \\
\hline$\geq 45$ & 84 & 23 & 61 & \\
\hline$<45$ & 52 & 17 & 35 & \\
\hline Gender & & & & 0.340 \\
\hline Male & 73 & 24 & 49 & \\
\hline Female & 63 & 16 & 47 & \\
\hline Extent of resection & & & & 0.634 \\
\hline Partial & 37 & 10 & 27 & \\
\hline Total & 99 & 30 & 69 & \\
\hline WHO grade & & & & $<0.001$ \\
\hline $\mathrm{I} / \mathrm{II}$ & 72 & 38 & 34 & \\
\hline III/IV & 64 & 2 & 62 & \\
\hline KPS score & & & & $<0.001$ \\
\hline$\geq 80$ & 76 & 36 & 40 & \\
\hline$<80$ & 60 & 4 & 56 & \\
\hline
\end{tabular}

c-Cbl, casitas B-lineage lymphoma; KPS, Karnofsky performance status; WHO, World Health Organization.

in Table I. Written informed consent was obtained from each patient enrolled in the study.

Immunohistochemistry. Samples were fixed in $10 \%$ formaldehyde solution (Santa Cruz Biotechnology, Inc., Dallas, TX, USA) and embedded in paraffin (Sigma-Aldrich, St. Louis, MO, USA) blocks, which were subsequently cut at a thickness of $4 \mu \mathrm{m}$. Sections were mounted on glass slides, deparaffinized in xylene and rehydrated in a graded series of alcohol (Sigma-Aldrich), followed by boiling in $10 \mathrm{mmol} / 1$ citrate buffer (Santa Cruz Biotechnology, Inc.) at pH 6.0 for 10 mins for antigen retrieval. Following inhibition of endogenous peroxidase activity by incubation in methanol containing $0.3 \%$ $\mathrm{H}_{2} \mathrm{O}_{2}$ (Santa Cruz Biotechnology, Inc.) for 30 min, sections were blocked with $2 \%$ bovine serum albumin (Sigma-Aldrich) for $30 \mathrm{~min}$ and incubated overnight at $4^{\circ} \mathrm{C}$ with rabbit anti-human c-Cbl monoclonal antibody (cat. no. ab32446; dilution, 1:200; Abcam, Cambridge, UK). Upon washing three times with phosphate-buffered saline, sections were incubated with horseradish peroxidase-conjugated mouse anti-rabbit immunoglobulin (Ig)G (cat. no. 2357; dilution; 1:200; Santa Cruz Biotechnology, Inc.) for $30 \mathrm{~min}$, followed by reaction with 3,3'-diaminobenzidine (Sigma-Aldrich) and counterstaining with hematoxylin (Abcam). For the negative controls, the primary antibody was substituted with a nonspecific rabbit IgG antibody (cat. no. sc-2027; Santa Cruz Biotechnology, Inc.). A Eclipse 90i microscope was used to view the sections (Nikon Corporation, Tokyo, Japan).

Immunoreactivity was evaluated and scored semi-quantitatively by two pathologists who were blinded to the patients' clinical data. Using the double scoring system (staining intensity multiplied by staining area), the staining intensity was evaluated as follows: 0 , no staining; 1 , definite but weak staining; 2 , moderate staining; and 3 , strong staining. The staining area was scored as follows: $1,<35 \%$ of cells were stained; $2,35-75 \%$ of cells were stained; and $3,>75 \%$ of cells were stained. High c-Cbl expression was defined as a score $\geq 4$, whereas low c-Cbl expression was defined as a score $<4$.

Western blot analysis. Whole cell lysates were prepared from glioma tissue, and western blotting was performed as previously described (15). Protein concentration was determined with the Protein Quantitation kit (Bradford assay; Abcam), using bovine serum albumin as a standard. Lysates $(20 \mathrm{mg})$ were solubilized in Laemmli sample buffer (Abcam) by boiling, and then resolved by $10 \%$ sodium dodecyl sulfate polyacrylamide gel electrophoresis, followed by electrotransfer (45V for $15 \mathrm{~h}$ ) onto a nitrocellulose membrane (Amersham; GE Healthcare Life Sciences, Chalfont, UK), which was then incubated with the rabbit anti-c-Cbl antibody at $4^{\circ} \mathrm{C}$ overnight, followed by incubation with the peroxidase-conjugated anti-rabbit $\mathrm{IgG}$ at room temperature for $1 \mathrm{~h}$. Rabbit anti-mouse monoclonal $\alpha$-tubulin (cat. no. ab52866; dilution, 1:2,000; Abcam) antibody served as a loading control. The immune complexes were visualized with an enhanced chemiluminescence western blotting detection system (Amersham; GE Healthcare Life Sciences). Quantity One 4.6 software (Bio-Rad Laboratories, Inc., Hercules, CA, USA) was used for desitometry analysis.

$R T-q P C R$. Total RNA was isolated from tissues using TRIzol reagent (Invitrogen; Thermo Fisher Scientific, Inc., Waltham, MA, USA), according to the manufacturer's protocol. RNA was reverse transcribed using SuperScript First-Strand Synthesis System (Invitrogen; Thermo Fisher Scientific, Inc.), and PCR amplification was performed using the following sets of sense and antisense primers: c-Cbl, sense 5'-CGCTAAAGA 
Table II. Cox regression model for multivariate analysis of glioma prognostic factors.

\begin{tabular}{lrrrrrrrr}
\hline & \multicolumn{3}{c}{ Overall survival } & & \multicolumn{3}{c}{ Progression-free survival } \\
\cline { 2 - 3 } Variable & HR & $95 \%$ CI & P-value & & HR & $95 \%$ CI & P-value \\
\hline Age $(\geq 45$ vs. $<45$ years) & 1.763 & $1.222-2.543$ & 0.002 & & 1.747 & $1.210-2.532$ & 0.003 \\
Gender (male vs. female) & 0.516 & $0.471-0.546$ & $<0.001$ & & 0.531 & $0.485-0.581$ & $<0.001$ \\
Extent of resection (partial vs. total) & 3.668 & $3.242-4.149$ & $<0.001$ & & 3.475 & $3.076-3.927$ & $<0.001$ \\
WHO grade (III/IV vs. I/II) & 8.842 & $7.827-9.989$ & $<0.001$ & & 10.247 & $9.009-11.655$ & $<0.001$ \\
KPS score (<80 vs. $\geq 80)$ & 0.984 & $0.905-1.069$ & & 0.701 & & 0.926 & $0.852-1.008$ & 0.067 \\
c-Cbl expression (high vs. low) & 4.923 & $3.163-7.662$ & $<0.001$ & & 6.181 & & $3.854-9.915$ & $<0.001$ \\
\hline
\end{tabular}

CI, confidence interval; HR, hazard ratio; c-Cbl, casitas B-lineage lymphoma; KPS, Karnofsky performance status; WHO, World Health Organization.

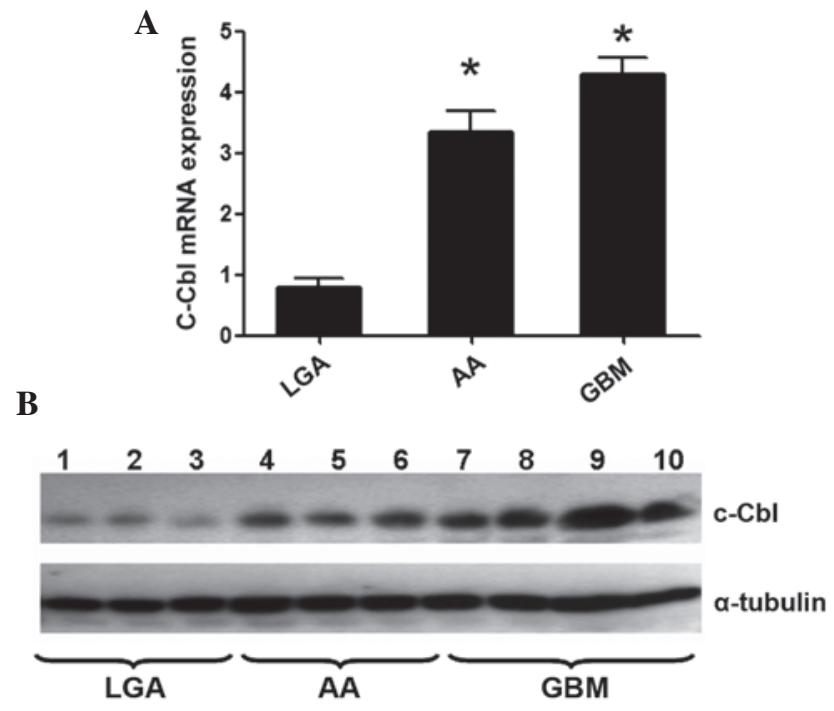

Figure 1. c-Cbl expression in glioma. (A) Higher messenger RNA expression levels of $\mathrm{c}-\mathrm{Cbl}$ were detected in high-grade (AA and gGBM) glioma samples, compared with low-grade (LGA) glioma samples, as determined by reverse transcription-quantitative polymerase chain reaction. The graph represents the expression levels of $\mathrm{c}-\mathrm{Cbl}$, relative to the expression levels of glyceraldehyde-3-phosphate dehydrogenase. ${ }^{~} \mathrm{P}<0.01$. (B) c-Cbl protein expression in LGA $(n=3)$, AA $(n=3)$ and GBM $(n=4)$ frozen samples, as determined by western blotting. $\alpha$-tubulin was used as a loading control. $\mathrm{c}-\mathrm{Cbl}$, casitas B-lineage lymphoma; AA, anaplastic astrocytoma; GBM, glioblastoma multiforme; LGA, low-grade astrocytoma; mRNA, messenger RNA.

ATAGCCCACCTTAT-3' and antisense 5'-ATGGCCTCC AGCCCAGAACTGAT-3'; and glyceraldehyde-3-phosphate dehydrogenase (GAPDH), sense 5'-TGCACCACCAACTGC TTAGC-3' and antisense 5'-GGCATGGACTGTGGTCAT GAG-3'. Primers were designed using the Primer premier software 5.0 (Premier Biosoft International, Palo Alto, CA, USA) and synthesized by Invitrogen (Thermo Fisher Scientific, Inc.). The amplification reaction consisted of 40 cycles of $94^{\circ} \mathrm{C}$ for $30 \mathrm{sec}, 60^{\circ} \mathrm{C}$ for $30 \mathrm{sec}$ and $72^{\circ} \mathrm{C}$ for $30 \mathrm{sec}$, and was conducted on an Applied Biosystems 7900HT Fast Real-Time PCR System (Thermo Fisher Scientific, Inc.) with $1.0 \mu \mathrm{l}$ of complementary DNA and SYBR Green Master Mix (Takara Bio, Inc., Otsu, Japan). RNA from 3 non-cancerous brain tissue samples, obtained from patients that had undergone surgery for drug-resistant temporal epilepsy at The First Hospital of
China Medical University, were used as the control. Data were collected and analyzed using SDS version 2.3 software (Applied Biosystems; Thermo Fisher Scientific, Inc.). The expression levels of the target gene were normalized to those of GAPDH, and determined using the $2^{-\Delta \Delta C q}$ method (16). The experiment was performed in triplicate, and repeated three times.

Statistical analysis. Statistical analysis was performed with SPSS version 19.0 (IBM SPSS, Armonk, NY, USA). The $\chi^{2}$ test was used to assess the association between c-Cbl expression and clinicopathological parameters. Patient survival curves were generated using the Kaplan-Meier method, and Cox regression analysis and log-rank test were used to identify independent prognostic factors. Data were expressed as the mean \pm standard deviation, and statistical significance was defined as a two-tailed $\mathrm{P}<0.05$.

\section{Results}

High c-Cbl expression is associated with clinicopathological features of glioma. RT-qPCR analysis of the messenger (m) RNA expression levels of $\mathrm{c}-\mathrm{Cbl}$ in high-grade [anaplastic astrocytoma (AA) and GBM] and low-grade [low-grade astrocytoma (LGA)] glioma revealed that the c-Cbl transcript was upregulated in high-grade glioma samples, compared with low-grade glioma samples $(\mathrm{P}<0.05$; Fig. 1A). Similarly, the protein levels of $\mathrm{c}-\mathrm{Cbl}$ were increased in AA and GBM, compared with LGA (Fig. 1B).

Immunohistochemical analysis demonstrated that $\mathrm{c}-\mathrm{Cbl}$ was mainly localized in the cytoplasm of malignant cells, and c-Cbl immunoreactivity was higher in high- vs. low-grade glioma (Fig. 2). The protein levels of c-Cbl were upregulated in 96 of $136(70.6 \%)$ patients with glioma, and high c-Cbl expression was correlated with World Health Organization (WHO) grade $(\mathrm{P}<0.001)$ and Karnofsky performance status (KPS) score $(\mathrm{P}<0.001)$. There were no significant differences in $\mathrm{c}-\mathrm{Cbl}$ expression with respect to gender, age or extent of resection $(\mathrm{P}>0.05$, Table I).

High c-Cbl protein expression levels are correlated with poor clinical outcome. Six patients were not available for follow-up, 


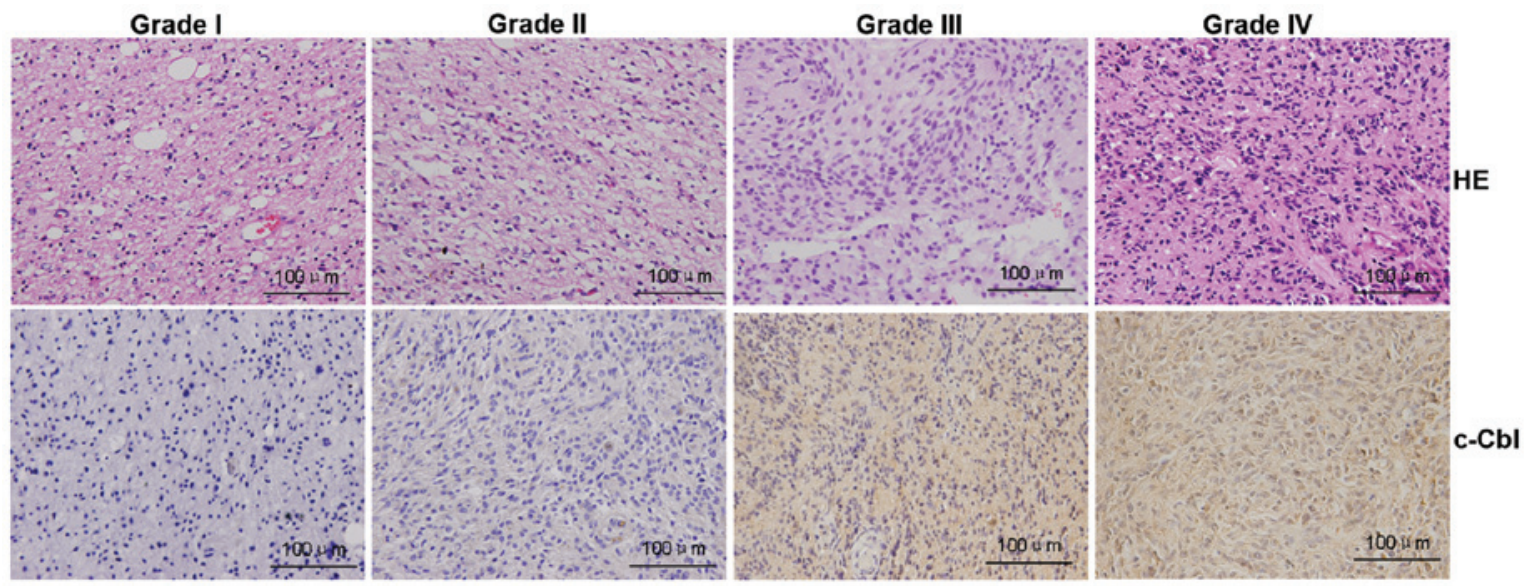

Figure 2. c-Cbl expression in glioma tissue samples, as determined by immunohistochemistry. Glioma specimens were counterstained with HE and labeled with an anti-c-Cbl antibody (magnification, x10). Glioma grades were assigned according to the World Health Organization criteria, as follows: I, pilocytic astrocytoma; II, diffuse astrocytoma (also called low-grade astrocytoma); III, anaplastic astrocytoma; and IV, glioblastoma. c-Cbl, casitas B-lineage lymphoma; HE, hematoxylin.

A

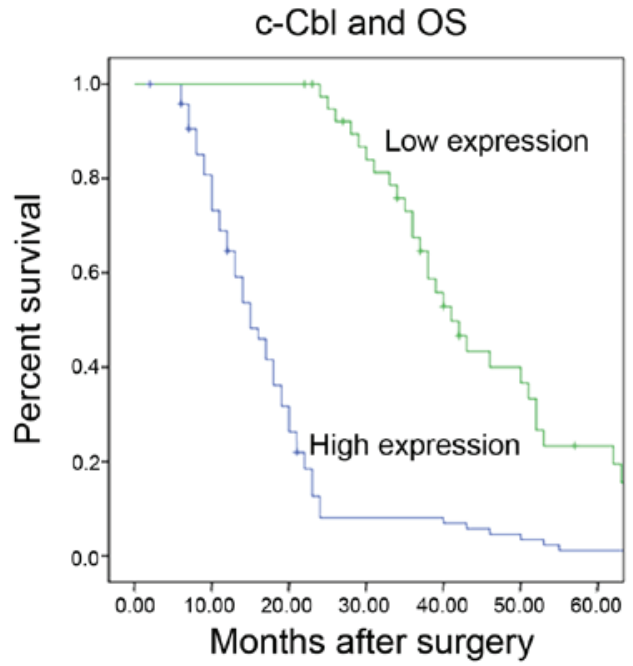

B

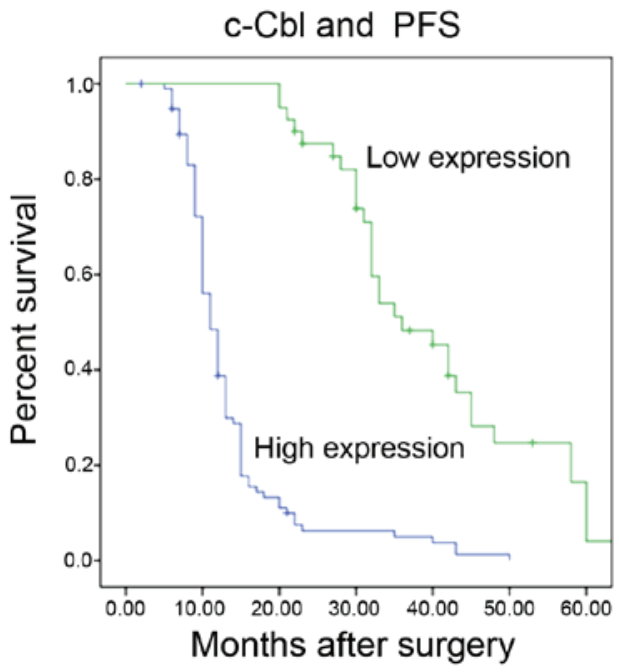

Figure 3. Kaplan-Meier survival analysis of patients with glioma as a function of c-Cbl expression. (A) Overall survival and (B) progression-free survival were decreased in patients with high expression levels of c-Cbl $(\mathrm{P}<0.001)$. c-Cbl, casitas B-lineage lymphoma; OS, overall survival; PFS, progression-free survival.

and therefore were excluded from the survival analyses. The remaining 130 patients were followed-up for 6-68 months. Multivariate analysis revealed that $\mathrm{c}-\mathrm{Cbl}$ expression was independently associated with overall survival [hazard ratio $(\mathrm{HR})=4.923$, 95\% confidence interval $(\mathrm{CI})=3.163-7.662$; $\mathrm{P}<0.001]$, and that $\mathrm{c}-\mathrm{Cbl}$ protein expression and $\mathrm{WHO}$ grade were independent prognostic factors of progression-free survival $(\mathrm{HR}=6.181,95 \% \mathrm{CI}=3.854-9.915 ; \mathrm{P}<0.001$, and $\mathrm{HR}=10.247,95 \% \mathrm{CI}=9.009-11.655 ; \mathrm{P}<0.001$, respectively) (Table II). The Kaplan-Meier analysis with log-rank test indicated that high $\mathrm{c}-\mathrm{Cbl}$ protein expression was associated with poor overall $(\mathrm{P}<0.001$; Fig. $3 \mathrm{~A})$ and progression-free survival $(\mathrm{P}<0.001$; Fig. 3B).

\section{Discussion}

The results of the current study indicate that glioma progression is associated with the upregulation of c-Cbl expression. Western blotting and immunohistochemical analysis of tissue samples derived from patients with glioma revealed a significant correlation between c-Cbl expression and WHO glioma grade. Furthermore, survival analysis demonstrated that overexpression of $\mathrm{c}-\mathrm{Cbl}$ is a predictor of poor prognosis in these patients.

$\mathrm{c}-\mathrm{Cbl}$ is a E3 ubiquitin ligase and multifunctional adaptor protein that regulates cell growth, invasion, apoptosis and angiogenesis in various human tumors, and mutations in the $\mathrm{c}-\mathrm{Cbl}$ gene may lead to tumorigenesis and metastasis in non-small cell lung cancer (14). In hematological malignancies, mutations in the genes of the $\mathrm{Cbl}$ family result in the failure of tyrosine kinase signaling of protein degradation, which is linked to poor prognosis (17). In previous studies, c-Cbl was expressed in $67 \%$ of gastric carcinoma cells, and was associated with the degree of tumor invasion and lymph node metastasis (11). c-Cbl was also demonstrated to promote tumor invasion (18). Furthermore, c-Cbl gene deficiency decreased osteoclast (19) and macrophage (20) migration, and modulated glioma cell invasion via regulation of matrix metalloproteinase 2 (21). 
In the present study, the protein levels of c-Cbl were significantly associated with WHO grade and KPS score, suggesting that $\mathrm{c}-\mathrm{Cbl}$ expression is associated with glioma development and progression. The present findings revealed that patients with higher c-Cbl expression in tumor tissue exhibited worse overall and progression-free survival than those expressing lower levels of the protein, indicating that $\mathrm{c}-\mathrm{Cbl}$ upregulation in glioma may increase tumor malignancy, and thereby lead to worse prognosis. Notably, the present results demonstrate that high levels of c-Cbl in human glioma tissues are associated with lower KPS scores and higher pathological grade. Survival analysis demonstrated that high c-Cbl expression in glioma tissues is correlated with, and is a prognostic factor for, lower progression-free and overall survival. A subgroup analysis suggested that $\mathrm{c}-\mathrm{Cbl}$ may be an independent prognostic factor for high (III and IV), but not low (I and II) histopathological grade. These findings demonstrate that the expression levels of $\mathrm{c}-\mathrm{Cbl}$ may be used to predict prognosis in patients with glioma following surgery.

The mechanisms underlying the oncogenic functions of c-Cbl in glioma remain to be investigated. A previous study reported a link between c-Cbl-mediated epidermal growth factor receptor signaling, tumor progression and metastasis, and poor prognosis in human gastric carcinoma (6). Identifying c-Cbl target mRNAs and binding partners will provide additional insight into the role of $\mathrm{c}-\mathrm{Cbl}$ in gliomagenesis.

Considering the poor prognosis of patients with glioma with the currently available therapies, the development of novel treatment approaches is necessary, which requires a better understanding of the pathophysiological and molecular properties of gliomas. The findings of the present study suggest that high $\mathrm{c}-\mathrm{Cbl}$ expression is a prognostic biomarker for glioma malignancy, and provide a basis for developing novel treatments.

\section{Acknowledgements}

The present study was supported by the National Natural Science Foundation of China (Beijing, China; grant nos. 81101917 and 81270036), and the Natural Science Foundation of Liaoning Province (Shenyang, China; grant no. 2013021045).

\section{References}

1. Ma R, de Pennington N, Hofer M, Blesing C and Stacey R: Diagnostic and prognostic markers in gliomas - an update. $\mathrm{Br}$ J Neurosurg 27: 311-315, 2013

2. Hentschel SJ and Lang FF: Current surgical management of glioblastoma. Cancer J 9: 113-125, 2003.

3. Diamond EL, Corner GW, De Rosa A, Breitbart W and Applebaum AJ: Prognostic awareness and communication of prognostic information in malignant glioma: A systematic review. J Neurooncol 119: 227-234, 2014

4. Clarke J, Butowski N and Chang S: Recent advances in therapy for glioblastoma. Arch Neurol 67: 279-283, 2010.
5. Andoniou CE, Thien CB and Langdon WY: Tumour induction by activated abl involves tyrosine phosphorylation of the product of the cbl oncogene. EMBO J 13: 4515-4523, 1994.

6. Thien CB and Langdon WY: Cbl: Many adaptations to regulate protein tyrosine kinases. Nat Rev Mol Cell Biol 2: 294-307, 2001.

7. Di Fiore PP and De Camilli P: Endocytosis and signaling. An inseparable partnership. Cell 106: 1-4, 2001.

8. Joazeiro CA and Hunter T: Biochemistry. Ubiquitination - more than two to tango. Science 289: 2061-2062, 2000.

9. Waterman $\mathrm{H}$ and Yarden Y: Molecular mechanisms underlying endocytosis and sorting of ErbB receptor tyrosine kinases. FEBS Lett 490: 142-152, 2001.

10. Mohapatra B, Ahmad G, Nadeau S, Zutshi N, An W, Scheffe S, Dong L, Feng D, Goetz B, Arya P, et al: Protein tyrosine kinase regulation by ubiquitination: Critical roles of Cbl-family ubiquitin ligases. Biochim Biophys Acta 1833: 122-139, 2013.

11. Ito R, Nakayama H, Yoshida K, Matsumura S, Oda N and Yasui W: Expression of Cbl linking with the epidermal growth factor receptor system is associated with tumor progression and poor prognosis of human gastric carcinoma. Virchows Arch 444: 324-331, 2004.

12. Cristóbal I, Manso R, Rincón R, Caramés C, Madoz-Gúrpide J, Rojo F and García-Foncillas J: Up-regulation of c-Cbl suggests its potential role as oncogene in primary colorectal cancer. Int J Colorectal Dis 29: 641, 2014.

13. Knight JF, Shepherd CJ, Rizzo S, Brewer D, Jhavar S, Dodson AR, Cooper CS, Eeles R, Falconer A, Kovacs G, et al: TEAD1 and c-Cbl are novel prostate basal cell markers that correlate with poor clinical outcome in prostate cancer. Br J Cancer 99: 1849-1858, 2008

14. Tan YH, Krishnaswamy S, Nandi S, Kanteti R, Vora S, Onel K, Hasina R, Lo FY, El-Hashani E, Cervantes G, et al: CBL is frequently altered in lung cancers: Its relationship to mutations in MET and EGFR tyrosine kinases. PLoS One 5: e8972, 2010, Hsu HH [added].

15. Shibuya N, Inoue K, Tanaka G, Akimoto K and Kubota K: Augmented pentose phosphate pathway plays critical roles in colorectal carcinomas. Oncology 88: 309-319, 2015.

16. Livak KJ and Schmittgen TD: Analysis of relative gene expression data using real-time quantitative PCR and the 2(-Delta Delta C(T)) Method. Methods 25: 402-408, 2001.

17. Makishima H, Cazzolli H, Szpurka H, Dunbar A, Tiu R, Huh J, Muramatsu H, O'Keefe C, Hsi E, Paquette RL, et al: Mutations of e3 ubiquitin ligase cbl family members constitute a novel common pathogenic lesion in myeloid malignancies. J Clin Oncol 27: 6109-6116, 2009.

18. Nam JM, Onodera Y, Mazaki Y, Miyoshi H, Hashimoto S and Sabe H: CIN85, a Cbl-interacting protein, is a component of AMAP1-mediated breast cancer invasion machinery. EMBO J 26: 647-656, 2007.

19. Chiusaroli R, Sanjay A, Henriksen K, Engsig MT, Horne WC, $\mathrm{Gu} \mathrm{H}$ and Baron R: Deletion of the gene encoding $\mathrm{c}-\mathrm{Cbl}$ alters the ability of osteoclasts to migrate, delaying resorption and ossification of cartilage during the development of long bones. Dev Biol 261: 537-547, 2003.

20. Caveggion E, Continolo S, Pixley FJ, Stanley ER, Bowtell DD, Lowell CA and Berton G: Expression and tyrosine phosphorylation of $\mathrm{Cbl}$ regulates macrophage chemokinetic and chemotactic movement. J Cell Physiol 195: 276-289, 2003.

21. Lee $\mathrm{H}$ and Tsygankov AY: c-Cbl regulates glioma invasion through matrix metalloproteinase 2. J Cell Biochem 111: 1169-1178, 2010. 